\title{
Understanding User Requirements in Take-Home Diabetes Management Technologies
}

\author{
Tom Owen \\ Future Interaction Technology Lab, \\ Swansea University, \\ Swansea, UK. \\ cstomo@swansea.ac.uk
}

\author{
George Buchanan \\ Centre for $\mathrm{HCl}$ Design, \\ City University, \\ London, UK. \\ george.buchanan.1@city.ac.uk
}

\author{
Harold Thimbleby \\ Future Interaction Technology Lab, \\ Swansea University, \\ Swansea, UK. \\ csharold@swansea.ac.uk
}

\begin{abstract}
People who suffer from Diabetes are required to make frequent decisions on their personal treatment based on results from glucose monitors. Yet the results returned from the devices form only a part of the decisionmaking process. We seek to understand the role that glucose monitors have in patient's management practices and how technology could support patients' management further. From a series of interviews, we arrive at the hypothesis that the capture of the contextual information will both aid the understanding of results, and allow for enhanced support during non-routine occurrences.
\end{abstract}

Healthcare, Medical device, Management practices, Contextual information

\section{INTRODUCTION}

There has been a recent trend towards patients becoming more involved in the management and treatment of their own conditions. Those required to be involved in maintaining their own conditions have a wealth of information and factors to process and understand. For example, those affected by Diabetes are faced with a constant challenge to maintain their glucose levels through tight management of food intake and insulin dosing. Devices such as glucose monitors form an essential part of this process by allowing patients to check the current level of glucose in their blood stream. This information can then be used to make future treatment decisions, such as adjusting insulin injection levels. However, the results that the monitors provide are the end result of the previous hours' activities. Important contextual information such as food consumed and exercise performed (whether planned or completed) are also involved in the patient's treatment decisions and condition management.

We aim to gain insight into the existing management practices and where the potential to support these practices exists and to achieve this we have conducted a series of interviews with people who have Diabetes. Our interviews were targeted primarily at understanding the management strategies when treating Diabetes and what challenges are faced.

\section{BACKGROUND AND MOTIVATION}

Research into the management practices of patients who are involved in monitoring their own conditions has focused on diseases such as Asthma (Yun et al. 2010) and Diabetes (Mamykina et al. 2006), (Mamykina and Mynatt 2007). It was discovered that patients often observe their own actions to enable the ability to spot potential areas where they could gain more control of their condition.

The interpretation of individual results has been established as a context-sensitive facet of a diabetes led life. Simply knowing a result is meaningless without knowing the situations that occurred around it. To provide a greater understanding of glucose results, one study (Smith et al. 2006) introduced photography as a means of capturing information to give context for glucose meters. Patients were asked to take pictures of the food they were eating around the time a test was performed. Users of the system were then able to upload data into a system colourcoded results (between low, normal and high) and allowed users to match food choices with resulting scores.

The contextual information is not only important to patients: clinicians also form a part of the management process. Providing information to clinicians can provide a platform for further education 
to patients regarding their diabetes management. Hela et al. (Hela et al. 2009) proposed a monitoring system for patients' houses that monitored daily events such as physical activity and times at which food has been consumed. From this data, clinicians will able to determine adherence to health plans set out to the patients and highlight areas which are not being followed.

The management practices of Diabetes patients are not limited to the home. To assist people to control their glucose, Kanstrup et al. (Kanstrup et al. 2010) developed "Living Laboratory" which provides a service to specify food contents at restaurants. Carbohydrate counting forms a key part of Diabetes treatment, as ultimately the carbohydrates will be broken down into glucose within the body. Therefore, knowing the contents of food that is served in restaurants will allow patients to adjust their insulin doses in light of the new information provided.

The existing, literature, then, suggests that context of lifestyle, psychology and recent events all play a role in how a patient interprets each reading. The broader concerns of self-image and management strategies also apply across sets of readings, and previous research in other conditions suggests that issues of doubt and motivation influence effective management. However, this body of knowledge does not yet give us sufficient insight to design and build predictably and provably effective tools that assist a patient's ongoing management of their condition. Indeed, the lack of successful final summative evaluation, suggests that this is very much an open research agenda.

Our goal is to extend research on the role of context in managing diabetes, to arrive at a better view of how different contexts interact, and contribute to effective strategy. To that end, we have conducted a study to help us achieve a more detailed insight into the existing management strategies employed by people with diabetes.

\section{STUDY}

In order to recruit participants for our study, we distributed emails amongst staff and students of a university. The email requested people who had Diabetes and made regular use of glucose monitors or if they knew someone who met the criteria. We also included a brief description of what the study would entail, such as; length of study and topics to be discussed (glucose monitoring habits and experiences). An incentive of a $£ 5$ gift voucher was offered for particpation.

\subsection{Procedure}

Before attending the interviews, participants were asked for a location that they would feel most comfortable completing the discussions in, personal offices and coffee shops were the preferred option by all participants. Given the potentially intrusive nature of the discussion (a participant's health condition) it was deemed that allowing the participants this option was most suitable.

Participants were presented with consent forms confirming their agreement to take part. The forms also had the additional use of outlining the study procedure and aims to the participants, which was also re-enforced verbally by the researcher conducting the interview. We requested 30 minutes of participants' time, but typically the interviews lasted for 20 minutes.

We used a semi-structured approach to our interview sessions to allow us to both systematically address the key issues suggested by the previous literature, and to explore in detail both other issues that appeared to be important for each interviewee, and also participants' own elaborations on our questions. Our focus was to understand how existing technology - particularly the glucose meter - played in the participants' self-management; how the interviewees decided and monitored where and when to check their glucose levels; how those strategies had emerged, what threats and changes disrupted them, and how they responded to, and managed, changes in their condition.

During the sessions extensive notes, as well as full audio recording were taken. The resulting audio was then fully transcribed for the purpose of in-depth analysis of the discussions, with the notes being used to highlight high points of interest. We then employed a general qualitative inductive method to analyse the notes taken. By reviewing each session and looking for similar comments between different participants, we discovered a series of themes.

\subsection{Participant Information}

Through our recruitment emails, 13 participants (seven male, six female) responded and attended the interviews with ages amongst the participants ranging from 19 - 65 (average age 46). Nine of our participants had Type 1 diabetes and four had Type 2 diabetes. The high proportion of Type 1s in our participant pool is likely to be a result of our request for people who make regular use of glucose monitors. We have elected to group both types to allow us to an understanding of the variety of strategies employed that are impacted by different situations. 


\section{EMERGING THEMES}

Upon completion of our study, several consistent themes emerged across the individual responses from the interviews. This section will outline the five most critical factors, which together cover the main elements of the participants' experiences.

\subsection{Monitoring}

Attitudes towards dealing with glucose results varied throughout our participants, P5 had particularly extreme views on his own condition "I have more pressing health issues, so I tend to ignore the diabetes, even though I know it will be a problem in years to come". Alternatively, P12 was extremely keen to log all aspects of her activity from glucose results, to food eaten and unusual situations that occurred. P12 noted that situations such as exams greatly impacted on her glucose levels, factors such as stress and adrenaline are known to alter the body chemistry sufficiently to impact blood glucose levels.

P2 stated that his monitoring intensified at particular times in the year, often for a week. These intensive monitoring spells were brought on by a personal desire for more information, or his partner's concern that P2's health may be worsening, and when GPs requested additional testing on the lead-up to a scheduled clinic visit. P10 made use of a 'tagging' feature that her monitor provided; she had the ability to mark results as pre-meal and post-meal. She was then able to upload her scores into a computer program which enabled her to compare different results with each other. Other participants appeared to only engaged in reflection of a sequence of results when they were returned unexpected results from their meters: P6 "If I can't decide why it was high, just keep an eye on it for a couple of days to see if recurring problem.

P12 described that moving onto a continuous insulin delivery therapy had greatly impacted on the frequency with which she monitored. She described how, when first using the continuous pump, tight glucose control was necessary, and this led to regular testing every 2-3 hours. P12 stated that they "Never really got out of the habit" of this intense regime. So, the demands of complying with clinical procedures, and the repetitive nature of this regime, leads to the formation of supporting habits.

\subsection{Habits}

The participants frequently described their own personal habits and rituals that are involved in, and emerge from, their management processes. P8 went to the extremes of tightly controlling the types of food he ate, portion sizes and times of the day at which he eats, "I don't get any surprises this way". Despite being offered by his clinic for a more flexible meal plan, after forming his own management style over 45 years he felt that his current style was predictable and he suffered fewer "surprising sugar results".

P3 also noticed changes in management style, stating that using injections led to a strict food schedule. Types of food eaten and the time at which they were consumed were based on the insulin injections that had previously been performed. Food intake was also a concern of P5, who had eliminated foods with high carb values as a method of controlling glucose levels.

Intensive monitoring was described by several of our participants, with P2 indicating that they had been recommended to use monitor less. P1 stated that "Monitoring is a bad way of controlling diabetes, this is responsive whereas it should already be controlled through good eating and exercise" and perhaps this is why P2 has been discouraged from monitoring as frequently. As stated in Section 1, glucose results are the end result of previous activity and purely dealing with glucose results is similar to dealing with a problem once it has already occurred, rather than preparing.

\subsection{Confidence}

When asked about whether they had a good idea of their glucose levels before performing a test, only participant P5 stated that he was rarely sure what his result would be before testing. The remaining twelve participants indicated that the majority of the time, they had a good idea of what the result would be. Performing glucose tests at specific points during the day has resulted with participants being in tune with how their body is feeling and translate that into glucose scores. It is even the case that the participants who took part in our study were comfortable making changes to their insulin doses and manipulate glucose levels in anticipation of future events. Such an example was described by P13, who discussed his approach to preparing for visits to the gym, indicating that to account for the body using up sugar during exercise, he would knowingly push up his glucose levels to prepare.

Yet this confidence in the control of their condition is only maintained providing 'normality' continues and there are several factors that can remove the confidence from participants. For instance, P2 has a consistent glucose 'spike' during late morning that is unexplained by both him and clinicians despite his attempts to determine the problem. He described this as a disappointment, a sentiment which was echoed by P4 "sometimes when I haven't eaten anything I shouldn't I still get a bad result and I get disappointed." Yet this participant could handle bad 
scores when she had eaten known bad foods, as this provided an explanation that she could attribute the scores to.

The breakdown of long-term habits would often cause participants to lose some of their confidence in managing their condition. P9 described attending a session aimed to improved glucose control, but with a different approach to what he had been used to. While he felt comfortable with the changes, he noticed stress amongst other attendees who were intimidated by performing new calculations to their insulin doses. He described that people who had Diabetes for several years were able to form their own management style and that the new approach would represent a major change in their daily monitoring and treatment. Concerns about insulin doses and calculations were also highlighted by $\mathrm{P} 4$ "I have problems with the insulin calculations, they scare me a bit in case I get them wrong."

\subsection{Unusual Situations}

Perhaps the main issue that affected participant management of their condition was when they were put into situations that differed from what they described as normal days. P1 indicated that "the weather can affect testing, humidity and heat give the same impression as a hypo so I perhaps test more frequently than is needed."

P7 experienced problems with glucose control during a walking holiday. In order to prepare for the holiday, P7 sought advice from her clinicians as to how best to alter her insulin treatment to account for the increased exercise from walking. Subsequently she was advised to halve basal doses and keep bolus doses as normal. Yet during the holiday P7 found that this style was not suitable for her and her sugar levels were frequently too low, as a result she experimented with her treatment until she found an optimal strategy. P7 expressed regret that she hadn't kept a record of what solution she found, as she had since forgotten it and would likely have to go through the same process during the next holiday.

One participant took great care to log unusual events, P12 stated that exams caused her great amount of nerves and stress, which in turn increased her adrenaline levels. This change in body reaction greatly affected her glucose scores and in order to account for the changes, P12 kept detailed records of what had been previously done to correct glucose levels to ensure that she did not lose her glucose control during these times as she could employ strategies that she had already created.

Therefore, variations, regular or occasional, occur within diabetes treatment management, and this leads to uncertainties that focus the patient's immediate attention on diagnosing and correcting erroneous glucose scores, before refining their management practices. Where patterns are established in the patient's mind reassurance is easier to find. However, when P7 faced a new situation, interpretation was more difficult, and she now has lost the knowledge that she had gained then. Such omissions to capture previous experience lead to feelings of regret and some anxiety about future episodes.

\subsection{Concerns}

Performing glucose tests in public was a worry for several of our participants, with P12 finding the greatest difficulty. Testing whilst in the company of friends and family was not viewed as a problem, but being in public would often cause her to hide her test as much as possible, usually performing inside a handbag to give the illusion of looking for something. Situations such as being in a lecture hall caused further problems, as the lecture theatres she attended had sloped seating, the people behind her could potentially see what she was doing leaving her feeling self-conscious. An additional factor that discouraged her from testing in public was people around her having bad reactions "lots of people will ask what I am doing and I tell them I have Diabetes, then they get a bit embarrassed and don't know what to say." Participants P2 "it is a fiddle" and P4 "people look at you" also shared concerns about performing glucose tests in public. This suggests that some participants may in fact prefer discreet and hidden interactions with their devices that are simple to perform in order to avoid standing out from the crowd.

P2 was one of the participants who took a high interest in his results and would regularly perform trend-spotting in previous results. Whilst this made him feel comfortable most of the time, he felt that too much testing is bad was having a negative impact on his health; "the constant worry about results can push up my blood pressure." High blood pressure is a general concern for everyone, but P2's worries were a result of the risks of high blood pressure being increased in those with diabetes and that the risk of complications from either condition are raised.

\section{DISCUSSION}

It became apparent during our interviews that monitoring would (almost) always be the starting point of our cycle, as it is the key factor in the management of their condition. Some of our participants also undertook more intensive monitoring, such as taking note of food intake and tracking levels in preparation for exercise. The 


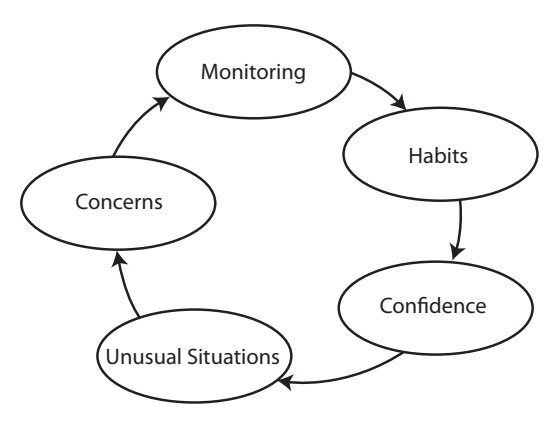

Figure 1: Diabetes Management Cycle.

repetition of everyday situations led to habits being formed, from testing at set times in a day through to having a rigid meal plan. These habits appeared to instil a feeling of confidence in participants, as they were in control of their sugar levels. This confidence now meant that they could deal with 'normal' days and events that would affect their glucose levels.

Examples were given of when habits became so routine that simple, yet fundamental errors in treatment were introduced. P11 discussed issues that arose when dealing with insulin dosing, specifically after exercise. They highlighted that there had been occasions when, after an exercise session, they would not take the exercise into account and give a 'normal' insulin dose. This dose proved to be an overdose, as the exercise had lowered the body's need for more insulin and the extra dose will have pushed the glucose levels below a desirable level.

Unusual situations outside the patient's normal routine would often produce unexpected glucose results and in turn, potentially unknown treatment requirements. Small factors - such as a particularly hot and humid day - could give the illusion of the body having low sugar levels, which would lead to more frequent testing as the usual safety barrier that participants had become accustomed to had been removed. Such exceptional circumstances undermined confidence, and led to a desire to explain and understand the situation. This often included referring back to previous events, and comparing to similar readings or contexts in the past.

Through every stage of the management cycle, some form of self-reflection occurs. The participants described situations at each of the five points of the cycle where some form of monitoring was being either recorded, or earlier situations and glycemic measures recalled. In turn, this information was used to judge and interpret the current context. This pervasive reflection shaped the future behaviours and progression through the cycle, with the most pointed need for evaluating the information occurring in the 'unusual situations' phase.
While (Mamykina et al. 2006) suggested that the decisions undertaken by people with diabetes were the result of monitoring and attributing changes to particular actions before modifying behaviour based on observations. We believe the management cycle has more factors involved. The data that we have collected indicates that there are two significant behaviours when reflecting on previous glucose scores. Results taken from ordinary days are often recorded and rarely checked back on. Instead the more intensive reflection occurs when unusual situations arise. These situations could be simple things such as illness up to more complex scenarios including holidays. (Li et al. 2011) suggested that there are phases of reflection; maintenance and discovery phases. We suggest that in our model, habits and confidence would fit in the maintenance phase, whereas unusual situations and concerns would present a shift into a discovery phase. The monitoring theme of our model could potentially fit both of $\mathrm{Li}$ et al.'s phases depending on what level of confidence or concern patients would be experiencing.

Li et al also suggest methods to support each of their two phases. The recommendations for supporting their discovery phase are particularly relevant to our research. The key suggestion for supporting discovery is to collect and retain as much data as possible at a low cost to the user. We now examine the requirements that can be extracted from our own data, building on the ideas of $\mathrm{Li}$ and others.

\subsection{Requirements for Self-Management}

One key constraint that emerged from the discussions was the need for privacy. Most of our participants noted a variety of issues that arose from conducting glucose tests in public. It is therefore important that any technology which aims to support diabetes management should be discreet and unobtrusive. Due to the extensive and ubiquitous nature of mobile phones, an everyday object that would not raise issue from surrounding people, they provide a suitable platform to facilitate and support the management process.

The key moments around unusual situations appear to be the crux of the management of most of our interviewees. Their interpretation of the situation requires a capture of recent and past context, and interpretation of both experience and knowledge. Logging, mental- and written-notes all contribute to this, but being systematic is a challenge.

Previous research, e.g. (Mamykina et al. 2010), has demonstrated the potential of using mobile phones to support 'reflective thinking' on the results of an individual's glucose testing. A key aim here was 
to support people with diabetes in their daily lives and the regular management of their self-treatment. In that research, data was gathered, and later reviewed by clinicians together with the patients, to facilitate a formal review of the patient's management strategy. Our research complements this approach, and draws some similar conclusions. However we focus on situations where reflection is done by the patient alone, where previous work has studied the situation where reflection is assisted by clinicians.

Those with diabetes need to reflect on events from some time previously, but this requires regular logging and other forms of context-capture. The original information capture is secondary to the diabetic's immediate management task, and must, consequently, be conducted with a minimum of effort. Extensive capture enhanced by the interpretative support of medical specialists can be helpful - but this may omit the contextual information that diabetics use to interpret their current circumstances. Making sense of various factors like place, time, and trends all need support.

\section{CONCLUSION \& FUTURE WORK}

It is possible that technology can provide a supportive role during this cycle to enable further logging of actions and reflection on the impact changes will have on glucose control. Having explored the possibility of employing mobile technology to fulfil this role and suggested a set of requirements that any system should adhere to. Our ultimate aim with this research is to understand what impact the addition of contextual information will have on patients' selfreflection and our proposed system will act as a platform to capture the context-factors that are an important aspect of the decision making process.

We hypothesize that immediate information on a mobile platform will have the potential to alleviate some of the emotional impact of perceived 'bad results,' such as results that do not match what patients anticipated before performing a test. There is likely to be an identifiable cause behind each undesirable result. The addition of contextual information may be able to capture several of these factors and allow for a better understanding of unexpected results. To pursue that goal, we aim to introduce a probe that provides ondemand contextual information to facilitate reflection. To assess the impact that mobile self-reflection could achieve, we aim to conduct a three month longitudinal diary study. Participants will be recruited and asked to make use of the mobile application that will capture data such as location throughout the day, pictures of experiences and exercise performed. It is hoped that the application will fit easily into the daily routines of people with diabetes and be inconspicuous rather than obtrusive.

\section{ACKNOWLEDGMENTS}

This work was generously supported by the EPSRC project EP/G059063/1.

\section{REFERENCES}

Hela, A., D. J. Cook, and M. Schmalz (2009). Smart home-based health platform for behavioral monitoring and alteration of diabetes patients. Journal of Diabetes Science and Technology 3, 141148.

Kanstrup, A. M., K. Bjerge, and J. E. Kristensen (2010). A living laboratory exploring mobile support for everyday life with diabetes. Wireless Personal Communications 53, 395-408.

Li, I., A. K. Dey, and J. Forlizzi (2011). Understanding my data, myself: supporting self-reflection with ubicomp technologies. In Proceedings of the 13th international conference on Ubiquitous computing, UbiComp '11, New York, NY, USA, pp. 405-414. ACM.

Mamykina, L., A. D. Miller, E. D. Mynatt, and D. Greenblatt (2010). Constructing identities through storytelling in diabetes management. In Proceedings of the 28th international Conference on Human Factors in Computing Systems, CHI '10, New York, NY, USA, pp. 1203-1212. ACM.

Mamykina, L. and E. D. Mynatt (2007). Investigating and supporting health management practices of individuals with diabetes. In Proceedings of the 1st ACM SIGMOBILE International Workshop on Systems and Networking Support for Healthcare and Assisted Living Environments, HealthNet '07, New York, NY, USA, pp. 49-54. ACM.

Mamykina, L., E. D. Mynatt, and D. R. Kaufman (2006). Investigating health management practices of individuals with diabetes. In Proceedings of the SIGCHI Conference on Human Factors in Computing Systems, CHI '06, New York, NY, USA, pp. 927-936. ACM.

Smith, B. K., J. Frost, M. Albayrak, and R. Sudhakar (2006, August). Integrating glucometers and digital photography as experience capture tools to enhance patient understanding and communication of diabetes self-management practices. Personal and Ubiquitous Computing 11(4), 273-286.

Yun, T.-J., H. Y. Jeong, H. R. Lee, R. Arriaga, and G. Abowd (2010). Assessing asthma management practices through in-home technology probes. In Pervasive Computing Technologies for Healthcare (PervasiveHealth), 2010. 